\title{
Slow motion of axisymmetric slip particles along their axes of revolution
}

\author{
Huan J. Keh *, Chia H. Huang \\ Department of Chemical Engineering, National Taiwan University, Taipei 106-17, Taiwan, ROC
}

Received 7 August 2003; received in revised form 7 January 2004; accepted 16 March 2004

Available online 20 July 2004

\begin{abstract}
This paper presents a combined analytical-numerical study for the Stokes flow caused by a rigid particle of revolution translating axisymmetrically in a viscous fluid. The fluid, which may be a slightly rarefied gas, is allowed to slip at the surface of the particle. A singularity method based on the principle of distribution of a set of Sampson spherical singularities along the axis of revolution within a prolate particle or on the fundamental plane within an oblate particle is used to find the general solution for the fluid velocity field that satisfies the boundary condition at infinity. The slip condition on the surface of the translating particle is then satisfied by applying a boundary-collocation technique to this general solution to determine the unknown coefficients. The drag force exerted on the particle by the fluid is evaluated with good convergence behavior for various cases. For the motion of a slip sphere, our drag results agree very well with the exact solution. For the translation of a no-slip spheroid, prolate or oblate, along its axis of symmetry, the agreement between our results and the analytical solutions obtained by using spheroidal coordinates is also quite good. It is found that the normalized drag force on the translating spheroid increases monotonically with an increase in the axial-to-radial aspect ratio of the spheroid for a no-slip or slightly-slip spheroid, and decreases monotonically as the ratio increases for a well-slip spheroid. The drag force on a spheroid with intermediate values of its slip coefficient is not a monotonic function of its aspect ratio. For a spheroid with a given aspect ratio, its drag force is a monotonically decreasing function of the slip coefficient of the particle.
\end{abstract}

(C) 2004 Elsevier Ltd. All rights reserved.

Keywords: Axisymmetric creeping flow; Aerosol particle of revolution; Spheroid; Slip surface; Drag force

\footnotetext{
${ }^{*}$ Corresponding author. Tel.: +886-2-2363-5462; fax: +886-2-2362-3040.

E-mail address: huan@ntu.edu.tw (H.J. Keh).
} 


\section{Introduction}

The movement of small particles in a continuous medium at low Reynolds numbers is of much fundamental and practical interest in the areas of chemical, biomedical, and environmental engineering and science. The theoretical treatment of this subject has grown out of the classic work of Stokes [1] for a translating rigid sphere in an unbounded viscous fluid. Oberbeck [2] extended this result to the translation of an ellipsoid. More recently, solutions of the creeping-flow problem have been obtained for particles which correspond to a coordinate surface of one of the special orthogonal coordinate systems in which the Stokes equations are simply separable $[3,4]$ and for long slender bodies [5,6]. Additionally, the low-Reynolds-number flow caused by the motion of a particle of more general shape has also been treated in the literature by the truncatedseries boundary-collocation method [7], the singularity method [8], and the boundary integral method [9-11].

When one tries to solve the Navier-Stokes equation, it is usually assumed that no slippage arises at the solid-fluid interfaces. Actually, this is an idealization of occurrence of the transport processes. The phenomenon that the adjacent fluid (especially if the fluid is a slightly rarefied gas) can slip frictionally over a solid surface has been confirmed, both experimentally and theoretically [12-14]. Presumably, any such slipping would be proportional to the local tangential stress next to the solid surface (see Eq. (7a)), at least as long as the velocity gradient is small $[15,16]$. The constant of proportionality, $\beta^{-1}$, may be termed a 'slip coefficient'. The quantity $\eta / \beta$ (where $\eta$ is the fluid viscosity) is a length, which can be pictured by noting that the fluid motion is the same as if the solid surface was displaced inward by a distance $\eta / \beta$ with the velocity gradient extending uniformly right up to no-slip velocity at the surface. Basset [15] has found that the drag force acting on a translating rigid sphere of radius $b$ with a slip-flow boundary condition at its surface (e.g., a settling aerosol sphere) is

$$
F=-6 \pi \eta b U \frac{\beta b+2 \eta}{\beta b+3 \eta}
$$

where $U$ is the translational velocity of the particle. When $\beta \rightarrow \infty$, there is no slip at the particle surface and Eq. (1) degenerates to the well-known Stokes law. In the limiting case of $\beta=0$, there is a perfect slip at the particle surface and the particle acts like a spherical invicid gas bubble.

In Eq. (1), the slip coefficient has been determined experimentally for various cases and found to agree with the general kinetic theory of gases. It can be evaluated from the relation

$$
\beta^{-1}=\frac{C_{m} l}{\eta},
$$

where $l$ is the mean free path of a gas molecule, and $C_{m}$ is a dimensionless constant of the gaskinetic slip, which is semi-empirically related to the momentum accommodation coefficient $f_{m}$ at the solid surface by $C_{m} \approx\left(2-f_{m}\right) / f_{m}$ [12]. Although $C_{m}$ surely depends upon the nature of the surface, examination of the experimental data suggests that it will be in the range 1.0-1.5 [17,18]. Note that the slip-flow boundary condition is not only applicable for a gas-solid surface in the continuum regime (Knudsen number $l / b \ll 1$ ), but also appears to be valid for some cases even 
into the molecular flow regime $(l / b \geqslant 1)$. The reciprocal of the factor $(\beta b+2 \eta) /(\beta b+3 \eta)$ in Eq. (1) is equivalent to the so-called Cunningham correction factor for the slip effect.

The slow motion of nonspherical particles with frictionally slip surfaces, such as pollen particles in gaseous media [19], was usually estimated by an adjusted sphere approximation [20]. This approximation comprises calculating the radius of the adjusted sphere which has the same slip correction factor as the nonspherical particle. Recently, the axisymmetric creeping flow of a viscous incompressible fluid past a spheroid which departs but little in shape from a sphere with the slip boundary condition was investigated, and an explicit expression for the drag force experienced by it was obtained to the first order in the small parameter characterizing the deformation [21,22]. However, the problem of the slow motion of nonspherical slip particles in a viscous fluid has not been exactly solved yet, mainly due to the fact that, if momentum slip is included, an analytic solution is not feasible for most orthogonal curvilinear coordinate systems, such as the prolate and oblate spheroidal coordinate systems [23,24]. In this paper we use a method of distributed internal singularities incorporated with a boundary-collocation technique to analyze the axisymmetric creeping flow generated by a slip particle of revolution translating along its axis of symmetry; the particle can be either prolate or oblate. The drag force exerted on the particle by the fluid as a function of the slip coefficient of the particle is calculated for various cases. For the special cases of a sphere and of a no-slip spheroid, our drag results show excellent agreement with the exact solutions. For the cases of a slip spheroid whose shape deviates slightly from that of a sphere, our results also agree quite well with the approximate analytical solution in the literature.

\section{Mathematical description of the problem}

We consider the translational motion of a general axisymmetric particle in an incompressible, Newtonian fluid along its axis of revolution at the steady state. The fluid may slip frictionally at the surface of the particle and is at rest at infinity. The circular cylindrical coordinates $(\rho, \phi, z)$ and spherical coordinates $(r, \theta, \phi)$ are utilized and the particle center is chosen to be the origin of the coordinates instantaneously, as shown in Fig. 1. The particle velocity equals $U \mathbf{e}_{z}$, where $\mathbf{e}_{z}$ is the unit vector in the positive $z$ direction. The Reynolds number is assumed to be sufficiently small so that the inertial terms in the fluid momentum equation can be neglected, in comparison with the viscous terms.

The fluid flow is governed by the Stokes equations,

$$
\begin{aligned}
& \eta \nabla^{2} \mathbf{v}-\nabla p=\mathbf{0}, \\
& \nabla \cdot \mathbf{v}=0,
\end{aligned}
$$

where $\mathbf{v}$ is the fluid velocity field and $p$ is the dynamic pressure distribution. Since the flow field is axially symmetric, it is convenient to introduce the Stokes stream function $\Psi(\rho, z)$ which satisfies Eq. (3b) and is related to the velocity components in cylindrical coordinates by 


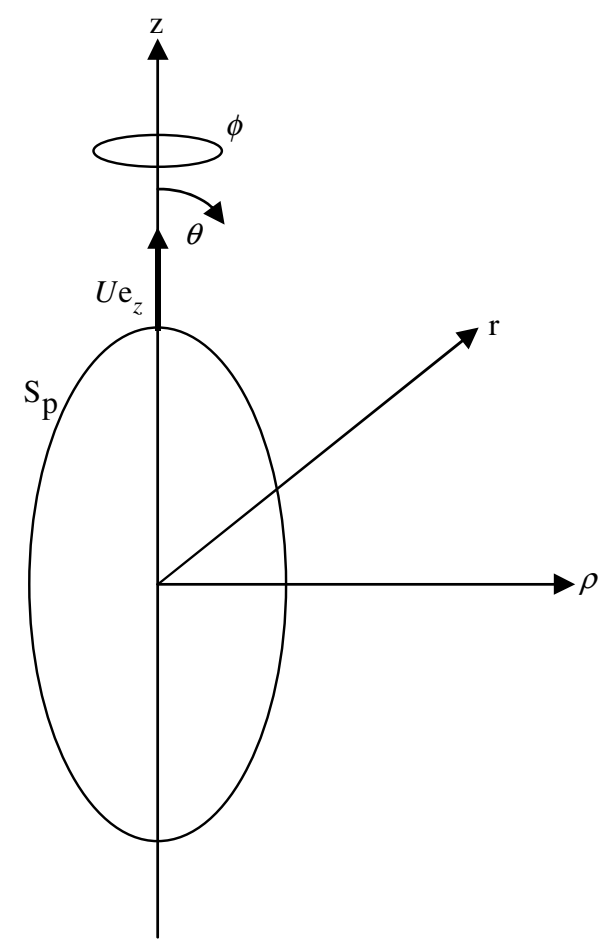

Fig. 1. Geometrical sketch for the motion of an arbitrary axisymmetric particle along its axis of revolution.

$$
\begin{aligned}
& v_{\rho}=\frac{1}{\rho} \frac{\partial \Psi}{\partial z}, \\
& v_{z}=-\frac{1}{\rho} \frac{\partial \Psi}{\partial \rho} .
\end{aligned}
$$

Taking the curl of Eq. (3a) and applying Eq. (4) gives a fourth-order linear partial differential equation for $\Psi$,

$$
E^{4} \Psi=E^{2}\left(E^{2} \Psi\right)=0
$$

where the axisymmetric Stokes operator $E^{2}$ is given by

$$
E^{2}=\rho \frac{\partial}{\partial \rho}\left(\frac{1}{\rho} \frac{\partial}{\partial \rho}\right)+\frac{\partial^{2}}{\partial z^{2}} .
$$

Since the relative tangential velocity of the fluid at the particle surface is proportional to the local tangential stress and the fluid is motionless far away from the particle, the boundary conditions are 


$$
\mathbf{v}=U \mathbf{e}_{z}+\frac{1}{\beta}(\mathbf{I}-\mathbf{n n}) \mathbf{n}: \tau \quad \text { on } S_{p}
$$

and

$$
\mathbf{v} \rightarrow \mathbf{0} \quad \text { as } r \rightarrow \infty
$$

Here, $\tau\left(=\eta\left[\nabla \mathbf{v}+(\nabla \mathbf{v})^{\mathrm{T}}\right]\right)$ is the viscous stress tensor, $1 / \beta$ is the frictional slip coefficient about the particle surface which is taken to be a constant, $\mathbf{I}$ is the unit dyadic, and $\mathbf{n}$ is the unit normal vector at the particle surface $S_{p}$ pointing into the fluid.

The drag force $F \mathbf{e}_{z}$ exerted by the fluid on the surface of the axisymmetric particle can be determined from [16]

$$
F=\frac{\eta}{2} \iint_{S_{p}} \rho^{2} \mathbf{n} \cdot \nabla\left(\frac{1}{\rho^{2}} E^{2} \Psi\right) \mathrm{d} S .
$$

To solve Eqs. (5)-(7), a set of Sampson spherical singularities (also called Sampsonlets) is chosen and distributed along the axis of revolution within a prolate particle [25] or on the fundamental plane within an oblate particle [26,27]. The flow field surrounding the particle is approximated by the superposition of the set of the spherical singularities and the boundary condition (7a) on the particle surface can be satisfied by making use of the multipole collocation method. For the special case of a spherical particle, only a single Sampsonlet which is placed at the particle center is needed.

The velocity components and stream function for the fluid motion caused by a Sampson spherical singularity at the point $\rho=0$ and $z=h$ are [28]

$$
\begin{aligned}
& v_{\rho}=\sum_{n=2}^{\infty}\left[B_{n} A_{1 n}(\rho, z-h)+D_{n} A_{2 n}(\rho, z-h)\right], \\
& v_{z}=\sum_{n=2}^{\infty}\left[B_{n} C_{1 n}(\rho, z-h)+D_{n} C_{2 n}(\rho, z-h)\right], \\
& \Psi=\sum_{n=2}^{\infty}\left[B_{n} E_{1 n}(\rho, z-h)+D_{n} E_{2 n}(\rho, z-h)\right],
\end{aligned}
$$

where $A_{i n}, C_{i n}$, and $E_{\text {in }}$ with $i=1$ and 2 are functions of position defined by Eq. (A.1) in Appendix A, and $B_{n}$ and $D_{n}$ are unknown constant coefficients. Note that the boundary condition (7b) is immediately satisfied by a solution in the form of Eq. (9).

In cylindrical coordinates, the boundary condition (7a) on the particle surface for the axisymmetric flow can be expressed as

$$
\left.\begin{array}{l}
v_{\rho}=\frac{1}{\beta}\left[\left(1-n_{\rho}^{2}\right) n_{\rho} \tau_{\rho \rho}-n_{\rho} n_{z}^{2} \tau_{z z}+\left(1-2 n_{\rho}^{2}\right) n_{z} \tau_{\rho z}\right] \\
v_{z}=U+\frac{1}{\beta}\left[\left(1-n_{z}^{2}\right) n_{z} \tau_{z z}-n_{z} n_{\rho}^{2} \tau_{\rho \rho}+\left(1-2 n_{z}^{2}\right) n_{\rho} \tau_{\rho z}\right]
\end{array}\right\} \text { on } S_{p}
$$


where $n_{\rho}$ and $n_{z}$ are the local $\rho$ and $z$ components of the unit normal vector $\mathbf{n}$. From Eq. (9a) and (9b), the components of the viscous stress tensor in Eq. (10) can be obtained as

$$
\begin{aligned}
\tau_{\rho \rho} & =\eta \sum_{n=2}^{\infty}\left[B_{n} \alpha_{1 n}(\rho, z-h)+D_{n} \alpha_{2 n}(\rho, z-h)\right], \\
\tau_{z z} & =\eta \sum_{n=2}^{\infty}\left[B_{n} \beta_{1 n}(\rho, z-h)+D_{n} \beta_{2 n}(\rho, z-h)\right], \\
\tau_{\rho z} & =\eta \sum_{n=2}^{\infty}\left[B_{n} \gamma_{1 n}(\rho, z-h)+D_{n} \gamma_{2 n}(\rho, z-h)\right],
\end{aligned}
$$

where $\alpha_{i n}, \beta_{i n}$, and $\gamma_{i n}$ with $i=1$ and 2 are functions of position defined by Eq. (A.2).

Eqs. (9) and (11) for the fluid flow and stress fields caused by a Sampsonlet and boundary condition (10) on the particle surface will be utilized in the following sections to solve for the fluid velocity induced by the axisymmetric motion of a particle of revolution.

\section{Solution for the motion of a spherical particle}

In this section a Sampson singularity described in the previous section is used to obtain the solution for the motion of a slip spherical particle of radius $b$. The drag results will be compared with the exact analytical solution given by Eq. (1).

The flow field generated by the translation of a sphere can be represented by a Sampsonlet placed at its center which is the origin of the coordinate frame. Thus, the velocity and stress components for the fluid motion caused by the sphere are given by Eqs. (9) and (11) with $h=0$. To determine the unknown constants $B_{n}$ and $D_{n}$, one can apply the boundary condition (10) at the particle surface to these velocity and stress components to yield

$$
\left.\begin{array}{l}
\sum_{n=2}^{\infty}\left[B_{n} A_{1 n}^{*}(\rho, z)+D_{n} A_{2 n}^{*}(\rho, z)\right]=0 \\
\sum_{n=2}^{\infty}\left[B_{n} C_{1 n}^{*}(\rho, z)+D_{n} C_{2 n}^{*}(\rho, z)\right]=U
\end{array}\right\} \quad(\text { at } r=b),
$$

where

$$
\begin{aligned}
& A_{i n}^{*}(\rho, z)=A_{\text {in }}(\rho, z)-\frac{\eta}{\beta}\left[\left(1-n_{\rho}^{2}\right) n_{\rho} \alpha_{i n}(\rho, z)-n_{\rho} n_{z}^{2} \beta_{i n}(\rho, z)+\left(1-2 n_{\rho}^{2}\right) n_{z} \gamma_{i n}(\rho, z)\right], \\
& C_{i n}^{*}(\rho, z)=C_{i n}(\rho, z)-\frac{\eta}{\beta}\left[\left(1-n_{z}^{2}\right) n_{z} \beta_{i n}(\rho, z)-n_{z} n_{\rho}^{2} \alpha_{i n}(\rho, z)+\left(1-2 n_{z}^{2}\right) n_{\rho} \gamma_{i n}(\rho, z)\right],
\end{aligned}
$$

and $i=1$ or 2 .

Substituting Eq. (9c) into Eq. (8) and applying orthogonality properties of the Gegenbauer polynomials, one can obtain a simple formula for the drag force exerted by the fluid on the particle,

$$
F=4 \pi \eta D_{2} .
$$


That is, only the first multipole of the Sampson singularity contributes to the hydrodynamic drag on the particle.

To satisfy the boundary condition (12) exactly along the entire semicircular generating arc of the sphere in a meridian plane would require the solution of the entire infinite array of unknown constants $B_{n}$ and $D_{n}$. However, the boundary-collocation technique [29,30] enforces the boundary condition at a finite number of discrete points on the particle's generating arc and truncates the infinite series in Eqs. (9), (11), and (12) into finite ones. The unknown constants in each term of the series permit one to satisfy the exact boundary condition at one discrete point on the particle surface. Thus, if the boundary is approximated by satisfying condition (12) at $N$ discrete points, then the infinite series are truncated after $N$ terms, resulting in a system of $2 N$ simultaneous linear algebraic equations in the truncated form of Eq. (12). This matrix equation can be solved by any of the standard matrix-reduction techniques to yield the $2 N$ unknown constants $B_{n}$ and $D_{n}$ required in the truncated equations for the flow field. The accuracy of the truncation technique can be improved to any degree by taking a sufficiently large value of $N$. Naturally, the truncation error vanishes as $N \rightarrow \infty$.

When specifying the points along the semicircular generating arc of the sphere where the boundary condition is exactly satisfied, the first point that should be chosen is $\theta=\pi / 2$, since this point defines the projected area of the particle normal to the direction of motion. In addition, the points $\theta=0$ and $\theta=\pi$ are also important. However, an examination of the system of linear algebraic equations for the unknown constants $B_{n}$ and $D_{n}$ shows that the coefficient matrix becomes singular if these points are used. To overcome the difficulty of singularity and to preserve the geometric symmetry of the spherical boundary about the equatorial plane $\theta=\pi / 2$, points at $\theta=\alpha, \pi / 2-\alpha, \pi / 2+\alpha$ and $\pi-\alpha$ are taken to be four basic collocation points [30]. Additional points along the boundary are selected as mirrorimage pairs about the plane $\theta=\pi / 2$ to divide the $\theta$ coordinate into equal parts. The optimum value of $\alpha$ in this work is found to be $0.01^{\circ}$, with which the numerical results of the drag force on the particle can converge to at least four significant figures. In principle, as long as the number of the collocation points is sufficiently large and the distribution of the collocation points is adequate, the solution of the drag force will converge and the shape of the particle can be well approximated, irrespective of the particle shape or boundary conditions. There are a few figures of streamline patterns for the axisymmetric motion of various particles obtained by using the boundary collocation method available in the literature [31,32], and they all show that the contour shape of $\Psi=0$ surface can approximate the shape of the particle very well with reasonable choices of the number and distribution of the collocation points.

In Table 1 , a number of numerical solutions of the dimensionless drag force $-F / 6 \pi \eta b U$ for the motion of a sphere are presented for various values of the slip parameter $\beta b / \eta$ (reciprocal of the dimensionless slip coefficient) using the collocation technique. All of the results were obtained by increasing the number of collocation points $N$ until the convergence of four significant digits is achieved. The exact solutions for $-F / 6 \pi \eta b U$ calculated using Eq. (1) are also listed in the bottom row of Table 1 for comparison. It can be seen that the results from the collocation method agree very well with the exact results for the desired accuracy and the rate of convergence is rapid. 
Table 1

Numerical results of the dimensionless drag force for a translating sphere with various values of the slip parameter

\begin{tabular}{lllll}
\hline$N$ & $-F / 6 \pi \eta b U$ & & & \\
\cline { 2 - 5 } & $\beta b / \eta \rightarrow \infty$ & $\beta b / \eta=10$ & $\beta b / \eta=1$ & $\beta b / \eta=0$ \\
\hline 4 & 1.0000 & 0.9231 & 0.7500 & 0.6667 \\
8 & 1.0000 & 0.9231 & 0.7500 & 0.6667 \\
Exact solution & 1.0000 & 0.9231 & 0.7500 & 0.6667 \\
\hline
\end{tabular}

\section{Axisymmetric motion of prolate particles}

We consider in this section the fluid motion caused by a general prolate axisymmetric particle translating along its axis of symmetry. The fluid may slip frictionally at the surface of the particle. A segment between points $\mathrm{A}\left(\rho=0, z=-c_{1}\right)$ and $\mathrm{B}\left(\rho=0, z=c_{2}\right)$ is taken along the axis of revolution inside the particle on which a set of Sampson spherical singularities are distributed $\left(c_{1}\right.$ and $c_{2}$ are positive constants). If the nose and the tail of the particle are round, then their centers of curvature can be chosen as A and B. The general solution of the flow field outside the particle can be constructed by the superposition of the Sampsonlets distributed on the segment AB, and Eqs. (9a) and (9b) are used to result in

$$
\left[\begin{array}{l}
v_{\rho} \\
v_{z}
\end{array}\right]=\sum_{n=2}^{\infty} \int_{-c_{1}}^{c_{2}}\left\{B_{n}(t)\left[\begin{array}{l}
A_{1 n}(\rho, z-t) \\
C_{1 n}(\rho, z-t)
\end{array}\right]+D_{n}(t)\left[\begin{array}{l}
A_{2 n}(\rho, z-t) \\
C_{2 n}(\rho, z-t)
\end{array}\right]\right\} \mathrm{d} t .
$$

The corresponding expressions for the components of the viscous stress tensor can be obtained using Eq. (11). Eq. (15) provides an exact solution for Eq. (3) that satisfies Eq. (7b), and the unknown density distribution functions for the singularities, $B_{n}(t)$ and $D_{n}(t)$, must be determined from the remaining boundary condition (7a) or (10) using the collocation technique. The drag force exerted by the fluid on the particle is obtained by the substitution of Eq. (15) into Eq. (8), with a result similar to Eq. (14),

$$
F=4 \pi \eta \int_{-c_{1}}^{c_{2}} D_{2}(t) \mathrm{d} t
$$

The density distribution functions $B_{n}(t)$ and $D_{n}(t)$ in Eq. (15) can be approximated to various orders of precision to satisfy the boundary condition at the particle surface. In this work we consider the constant-distribution, linear-distribution, and quadratic-distribution approximations.

\subsection{Constant density distribution}

In the constant density approximation, the segment $\mathrm{AB}$ is divided into $M$ small segments and the density distribution functions in each small segment are substituted by constants. Let $t_{m-1}$ and $t_{m}$ denote the coordinates of the two end points for the $m$ th segment, then one has $t_{0}=-c_{1}$ and $t_{M}=c_{2}$. If the length of each segment is the same, then $t_{j}=-c_{1}+j\left(c_{1}+c_{2}\right) / M$ for 
$j=1,2, \ldots, M-1$. In order to use the multipole collocation technique to satisfy the boundary condition at the particle surface, the infinite series in Eq. (15) are furthermore truncated after $N$ terms. With these arrangements Eq. (15) becomes

$$
\left[\begin{array}{c}
v_{\rho} \\
v_{z}
\end{array}\right]=\sum_{m=1}^{M} \sum_{n=2}^{N+1}\left\{B_{n m}\left[\begin{array}{l}
A_{1 n m 1}(\rho, z) \\
C_{1 n m 1}(\rho, z)
\end{array}\right]+D_{n m}\left[\begin{array}{l}
A_{2 n m 1}(\rho, z) \\
C_{2 n m 1}(\rho, z)
\end{array}\right]\right\}
$$

where the functions $A_{\text {inm } 1}$ and $C_{\text {inm } 1}$ with $i=1$ and 2 are defined by Eq. (A.8) in Appendix A and $B_{n m}$ and $D_{n m}$ are unknown density constants. Accordingly, the corresponding stress components can be written as

$$
\left[\begin{array}{c}
\tau_{\rho \rho} \\
\tau_{z z} \\
\tau_{\rho z}
\end{array}\right]=\eta \sum_{m=1}^{M} \sum_{n=2}^{N+1}\left\{B_{n m}\left[\begin{array}{l}
\alpha_{1 n m 1}(\rho, z) \\
\beta_{1 n m 1}(\rho, z) \\
\gamma_{1 n m 1}(\rho, z)
\end{array}\right]+D_{n m}\left[\begin{array}{l}
\alpha_{2 n m 1}(\rho, z) \\
\beta_{2 n m 1}(\rho, z) \\
\gamma_{2 n m 1}(\rho, z)
\end{array}\right]\right\}
$$

where the functions $\alpha_{i n m 1}, \beta_{i n m 1}$, and $\gamma_{i n m 1}$ with $i=1$ and 2 are also defined by Eq. (A.8).

Application of the boundary condition (7a) or (10) to Eqs. (17) and (18) yields

$$
\sum_{m=1}^{M} \sum_{n=2}^{N+1}\left\{B_{n m}\left[\begin{array}{l}
A_{1 n m 1}^{*}(\rho, z) \\
C_{1 n m 1}^{*}(\rho, z)
\end{array}\right]+D_{n m}\left[\begin{array}{c}
A_{2 n m 1}^{*}(\rho, z) \\
C_{2 n m 1}^{*}(\rho, z)
\end{array}\right]\right\}=\left[\begin{array}{c}
0 \\
U
\end{array}\right] \quad \text { on } S_{p},
$$

where the functions $A_{i n m 1}^{*}$ and $C_{i n m 1}^{*}$ with $i=1$ and 2 are given by Eq. (13) with the subscript in of its functions replaced by inm 1 . The collocation method allows the particle's boundary to be approximated by satisfying Eq. (19) at $M N$ discrete values of $z$ (rings) on its surface. This results in a set of $2 M N$ simultaneous linear algebraic equations, which can be solved by any matrixreduction technique to yield the $2 M N$ density constants $B_{n m}$ and $D_{n m}$ required in Eq. (17) for the fluid velocity field. Once these constants are determined, the drag force exerted by the fluid on the particle can be obtained from Eq. (16), with the result

$$
F=4 \pi \eta \sum_{m=1}^{M} D_{2 m}\left(t_{m}-t_{m-1}\right)
$$

\subsection{Linear density distribution}

The segment $\mathrm{AB}$ is divided into $M$ small segments, as we did in the previous subsection. However, the density functions in the $m$ th segment are now replaced by linear functions,

$$
\left[\begin{array}{c}
B_{n}(t) \\
D_{n}(t)
\end{array}\right]=\frac{t-t_{m-1}}{t_{m}-t_{m-1}}\left[\begin{array}{c}
B_{n m} \\
D_{n m}
\end{array}\right]+\frac{t_{m}-t}{t_{m}-t_{m-1}}\left[\begin{array}{c}
B_{n(m-1)} \\
D_{n(m-1)}
\end{array}\right] \text { for } t_{m-1} \leqslant t \leqslant t_{m} .
$$

Here $t_{m-1}$ and $t_{m}$ are the coordinates of the end points of the segment, and $B_{n(m-1)}, D_{n(m-1)}, B_{n m}$, and $D_{n m}$ are the corresponding density constants at these points. 
Substituting Eq. (21) into Eq. (15) for the fluid flow field and truncating the infinite series after $N$ terms, one obtains

$$
\begin{gathered}
{\left[\begin{array}{c}
v_{\rho} \\
v_{z}
\end{array}\right]=\sum_{m=1}^{M} \sum_{n=2}^{N+1}\left\{B_{n(m-1)}\left[\begin{array}{l}
V_{1 n m 1}(\rho, z) \\
W_{1 n m 1}(\rho, z)
\end{array}\right]+B_{n m}\left[\begin{array}{l}
V_{1 n m 2}(\rho, z) \\
W_{1 n m 2}(\rho, z)
\end{array}\right]+D_{n(m-1)}\left[\begin{array}{c}
V_{2 n m 1}(\rho, z) \\
W_{2 n m 1}(\rho, z)
\end{array}\right]\right.} \\
\left.+D_{n m}\left[\begin{array}{l}
V_{2 n m 2}(\rho, z) \\
W_{2 n m 2}(\rho, z)
\end{array}\right]\right\},
\end{gathered}
$$

and the corresponding stress components are

$$
\begin{gathered}
{\left[\begin{array}{c}
\tau_{\rho \rho} \\
\tau_{z z} \\
\tau_{\rho z}
\end{array}\right]=\eta \sum_{m=1}^{M} \sum_{n=2}^{N+1}\left\{B_{n(m-1)}\left[\begin{array}{c}
R_{1 n m 1}(\rho, z) \\
S_{1 n m 1}(\rho, z) \\
T_{1 n m 1}(\rho, z)
\end{array}\right]+B_{n m}\left[\begin{array}{c}
R_{1 n m 2}(\rho, z) \\
S_{1 n m 2}(\rho, z) \\
T_{1 n m 2}(\rho, z)
\end{array}\right]+D_{n(m-1)}\left[\begin{array}{c}
R_{2 n m 1}(\rho, z) \\
S_{2 n m 1}(\rho, z) \\
T_{2 n m 1}(\rho, z)
\end{array}\right]\right.} \\
\left.+D_{n m}\left[\begin{array}{l}
R_{2 n m 2}(\rho, z) \\
S_{2 n m 2}(\rho, z) \\
T_{2 n m 2}(\rho, z)
\end{array}\right]\right\}
\end{gathered}
$$

where the functions $V_{\text {inmk }}, W_{\text {inmk }}, R_{\text {inmk }}, S_{\text {inmk }}$, and $T_{\text {inmk }}$ with $i$ and $k$ equal to 1 and 2 are defined by Eqs. (A.3) and (A.4) in Appendix A. Application of the boundary condition (7a) or (10) to Eqs. (22) and (23) can be accomplished by utilizing the collocation technique. On the particle surface, Eqs. (10), (22) and (23) are applied at $(M+1) N$ discrete values of $z$ (rings). This generates a set of $2(M+1) N$ linear algebraic equations for the $2(M+1) N$ unknown constants $B_{n m}$ and $D_{n m}$. The fluid velocity field is completely solved once these coefficients are determined.

The drag force exerted by the fluid on the particle can be determined by Eq. (16) with the substitution of $D_{2}(t)$ expressed by Eq. (21). The result is

$$
F=2 \pi \eta \sum_{m=1}^{M}\left[D_{2(m-1)}+D_{2 m}\right]\left(t_{m}-t_{m-1}\right) .
$$

\subsection{Quadratic density distribution}

Similar to in Sections 4.1 and 4.2, the segment $\mathrm{AB}$ is again divided into $M$ small segments. In the quadratic density approximation, two end points and one middle point are taken to express the density distribution functions in each segment. Thus,

$$
\begin{aligned}
{\left[\begin{array}{c}
B_{n}(t) \\
D_{n}(t)
\end{array}\right]=} & \frac{\left(t-t_{m-1}\right)\left(t-\bar{t}_{m}\right)}{\left(t_{m}-t_{m-1}\right)\left(t_{m}-\bar{t}_{m}\right)}\left[\begin{array}{l}
B_{n(2 m)} \\
D_{n(2 m)}
\end{array}\right]+\frac{\left(t-t_{m}\right)\left(t-t_{m-1}\right)}{\left(\bar{t}_{m}-t_{m}\right)\left(\bar{t}_{m}-t_{m-1}\right)}\left[\begin{array}{l}
B_{(2 m-1)} \\
D_{(2 m-1)}
\end{array}\right] \\
& +\frac{\left(t-t_{m}\right)\left(t-\bar{t}_{m}\right)}{\left(t_{m-1}-t_{m}\right)\left(t_{m-1}-\bar{t}_{m}\right)}\left[\begin{array}{c}
B_{n(2 m-2)} \\
D_{n(2 m-2)}
\end{array}\right] \text { for } t_{m-1} \leqslant t \leqslant t_{m} .
\end{aligned}
$$

Here $\bar{t}_{m}=\left(t_{m-1}+t_{m}\right) / 2$ is the coordinate of the center of the $m$ th segment, and $B_{n(2 m-2)}, D_{n(2 m-2)}$, $B_{n(2 m-1)}, D_{n(2 m-1)}, B_{n(2 m)}$, and $D_{n(2 m)}$ are the corresponding density constants at the two end points and the middle point of the segment. 
Substituting Eq. (25) into Eq. (15) and truncating the infinite series after $N$ terms, one obtains

$$
\begin{aligned}
& {\left[\begin{array}{c}
v_{\rho} \\
v_{z}
\end{array}\right]=\sum_{m=1}^{M} \sum_{n=2}^{N+1}\left\{B_{n(2 m-2)}\left[\begin{array}{c}
V_{1 n m 1}^{\prime}(\rho, z) \\
W_{1 n m 1}^{\prime}(\rho, z)
\end{array}\right]+B_{n(2 m-1)}\left[\begin{array}{c}
V_{1 n m 2}^{\prime}(\rho, z) \\
W_{1 n m 2}^{\prime}(\rho, z)
\end{array}\right]+B_{n(2 m)}\left[\begin{array}{c}
V_{1 n m 3}^{\prime}(\rho, z) \\
W_{1 n m 3}^{\prime}(\rho, z)
\end{array}\right]\right.} \\
& \left.+D_{n(2 m-2)}\left[\begin{array}{c}
V_{2 n m 1}^{\prime}(\rho, z) \\
W_{2 n m 1}^{\prime}(\rho, z)
\end{array}\right]+D_{n(2 m-1)}\left[\begin{array}{c}
V_{2 n m 2}^{\prime}(\rho, z) \\
W_{2 n m 2}^{\prime}(\rho, z)
\end{array}\right]+D_{n(2 m)}\left[\begin{array}{c}
V_{2 n m 3}^{\prime}(\rho, z) \\
W_{2 n m 3}^{\prime}(\rho, z)
\end{array}\right]\right\}, \\
& {\left[\begin{array}{c}
\tau_{\rho \rho} \\
\tau_{z z} \\
\tau_{\rho z}
\end{array}\right]=\eta \sum_{m=1}^{M} \sum_{n=2}^{N+1}\left\{B_{n(2 m-2)}\left[\begin{array}{c}
R_{1 n m 1}^{\prime}(\rho, z) \\
S_{1 n m 1}^{\prime}(\rho, z) \\
T_{1 n m 1}^{\prime}(\rho, z)
\end{array}\right]+B_{n(2 m-1)}\left[\begin{array}{c}
R_{1 n m 2}^{\prime}(\rho, z) \\
S_{1 n m 2}^{\prime}(\rho, z) \\
T_{1 n m 2}^{\prime}(\rho, z)
\end{array}\right]\right.} \\
& +B_{n(2 m)}\left[\begin{array}{l}
R_{1 n m 3}^{\prime}(\rho, z) \\
S_{1 n m 3}^{\prime}(\rho, z) \\
T_{1 n m 3}^{\prime}(\rho, z)
\end{array}\right]+D_{n(2 m-2)}\left[\begin{array}{c}
R_{2 n m 1}^{\prime}(\rho, z) \\
S_{2 n m 1}^{\prime}(\rho, z) \\
T_{2 n m 1}^{\prime}(\rho, z)
\end{array}\right]+D_{n(2 m-1)}\left[\begin{array}{c}
R_{2 n m 2}^{\prime}(\rho, z) \\
S_{2 n m 2}^{\prime}(\rho, z) \\
T_{2 n m 2}^{\prime}(\rho, z)
\end{array}\right] \\
& \left.+D_{n(2 m)}\left[\begin{array}{c}
R_{2 n m 3}^{\prime}(\rho, z) \\
S_{2 n m 3}^{\prime}(\rho, z) \\
T_{2 n m 3}^{\prime}(\rho, z)
\end{array}\right]\right\}
\end{aligned}
$$

where the functions $V_{\text {inmk }}^{\prime}, W_{\text {inmk }}^{\prime}, R_{\text {inmk }}^{\prime}, S_{\text {inmk }}^{\prime}$, and $T_{\text {inmk }}^{\prime}$ with $i=1,2$ and $k=1,2,3$ are defined by Eqs. (A.5)-(A.7). To use the collocation technique, the boundary condition (10) after the substitution of Eqs. (26) and (27) is applied at $(2 M+1) N$ discrete values of $z$ along the surface of the particle. The resulting system of $2(2 M+1) N$ linear algebraic equations can be solved to yield the $2(2 M+1) N$ unknown constants $B_{n m}$ and $D_{n m}$ required in Eq. (26) for the fluid velocity components.

Substituting $D_{2}(t)$ given by Eq. (25) into Eq. (16), the drag force exerted on the particle is obtained as

$$
F=\frac{2}{3} \pi \eta \sum_{m=1}^{M}\left[D_{2(2 m-2)}+4 D_{2(2 m-1)}+D_{2(2 m)}\right]\left(t_{m}-t_{m-1}\right) .
$$

Note that the integrals in the functions $A_{\text {inmk }}, C_{\text {inmk }}, \alpha_{\text {inmk }}, \beta_{\text {inmk }}$, and $\gamma_{\text {inmk }}$ defined by Eq. (A.8) can be evaluated analytically. If numerical integrations are used for the evaluation of these functions, the accuracy and convergence behavior of the solution of drag force given by Eqs. (20), (24) and (28) depends on the precision of these numerical integrations.

Obviously, we can also use a cubic-distribution approximation for $B_{n}(t)$ and $D_{n}(t)$ in each small segment. But its relevant mathematical formulas would be quite lengthy.

\section{Solution for the motion of a prolate spheroidal particle}

In this section the method presented in the previous section is used to obtain the solution for the axisymmetric motion of a slip prolate spheroid. The surface of the prolate spheroid is represented in cylindrical coordinates by 


$$
z(\rho)= \pm a\left[1-\left(\frac{\rho}{b}\right)^{2}\right]^{1 / 2}
$$

where $a>b>0$ and $0 \leqslant \rho \leqslant b$ ( $a$ and $b$ are the major and minor semi-axes, respectively, of the prolate spheroid).

For a prolate spheroidal particle with a no-slip surface $(\beta b / \eta \rightarrow \infty)$ translating with a velocity $U$ along its axis of revolution in an unbounded fluid, the exact solution for the drag force exerted on the particle by the fluid is [16]

$$
F_{\infty}=-6 \pi \eta b U f_{\infty}
$$

with

$$
f_{\infty}=\left\{\frac{3}{4}\left(\zeta^{2}-1\right)^{1 / 2}\left[\left(\zeta^{2}+1\right) \operatorname{coth}^{-1} \zeta-\zeta\right]\right\}^{-1} .
$$

In Eq. (30b), $\zeta=a / c$ and $c=\left(a^{2}-b^{2}\right)^{1 / 2}$, which is the half distance between the two foci of the prolate spheroid.

In Section 3, collocation solutions for the translational motion of a spherical particle with a slip surface were presented and shown to be in perfect agreement with the exact solution. We now use the same collocation scheme incorporated with the method of distribution of Sampson singularities to obtain the corresponding solution for a slip prolate spheroid. In Table 2, numerical results of the nondimensional drag force $-F / 6 \pi \eta b U$ for the axisymmetric motion of a prolate spheroid are presented for three representative cases of the aspect ratio $a / b$ with various values of the slip parameter $\beta b / \eta$. The values of $-F / 6 \pi \eta b U$ are computed by applying either the linear (for $a / b=1.1$ and 2 ) or the quadratic (for $a / b=5$ ) density distribution at each segment for different $N$ and $M$ in Eqs. (22) and (26) (which shows convergence tests). For a spheroid with its aspect ratio close to unity, a constant density distribution of Sampsonlets can usually achieve good convergence behavior for the calculation of $F$. However, when the aspect ratio of the spheroid deviates further from unity (especially for the case of small values of $\beta b / \eta$ ), the convergence of the constant density approximation becomes poorer and higher-order approximations should be adopted. The exact solution of $f_{\infty}$ for the axisymmetric motion of a no-slip prolate spheroid (with $\beta b / \eta \rightarrow \infty$ ) given by Eq. (30b) is also given in Table 2 for comparison. It can be seen that our results from the method of distributed Sampson singularities agree very well with the exact solution in this limit. In general, the convergence behavior of the method of Sampsonlets is quite good, except for the case of large aspect ratio and small slip parameter.

Palaniappan [21] and Ramkissoon [22] investigated the problem of slow axisymmetric flow of a viscous incompressible fluid past a slip spheroid whose shape deviates slightly from that of a sphere. Their analytical result corresponding to the drag force exerted on the particle defined by Eq. (8), which is correct to the first order in the small parameter $\varepsilon$ characterizing the deformation, can be expressed as

$$
F=-6 \pi \eta b U\left[\frac{\beta b+2 \eta}{\beta b+3 \eta}-\varepsilon \frac{(\beta b)^{2}+6 \eta \beta b+6 \eta^{2}}{5(\beta b+3 \eta)^{2}}\right],
$$


Table 2

Numerical results of the dimensionless drag force for the motion of a prolate spheroid along its axis of revolution for various values of the aspect ratio and slip parameter of the spheroid

\begin{tabular}{|c|c|c|c|c|c|}
\hline \multirow[t]{2}{*}{$\beta b / \eta$} & \multirow[t]{2}{*}{$M$} & \multirow[t]{2}{*}{$N$} & \multicolumn{3}{|l|}{$-F / 6 \pi \eta b U$} \\
\hline & & & $a / b=1.1$ & $a / b=2$ & $a / b=5$ \\
\hline \multirow[t]{4}{*}{$\infty$} & 3 & 3 & 1.0201 & 1.2039 & 1.7848 \\
\hline & 4 & & 1.0201 & 1.2039 & 1.7848 \\
\hline & 5 & & 1.0201 & 1.2039 & 1.7848 \\
\hline & Exa & & 1.0201 & 1.2039 & 1.7848 \\
\hline \multirow[t]{5}{*}{10} & 4 & 3 & 0.9415 & 1.1163 & 1.6853 \\
\hline & 5 & & 0.9415 & 1.1163 & 1.6764 \\
\hline & 6 & & 0.9415 & 1.1163 & 1.6783 \\
\hline & 7 & & & & 1.6783 \\
\hline & Ap & tion & 0.9427 & 1.1195 & 1.7089 \\
\hline \multirow[t]{5}{*}{1} & 4 & 3 & 0.7520 & 0.8141 & 1.1833 \\
\hline & 5 & & 0.7520 & 0.8141 & 1.1731 \\
\hline & 6 & & 0.7520 & 0.8141 & 1.1749 \\
\hline & 7 & & & & 1.1750 \\
\hline & Ap & & 0.7662 & 0.9125 & 1.4000 \\
\hline \multirow[t]{5}{*}{0} & 4 & 3 & 0.6537 & 0.5635 & 0.3718 \\
\hline & 5 & & 0.6537 & 0.5635 & 0.3613 \\
\hline & 6 & & 0.6537 & 0.5635 & 0.3992 \\
\hline & 7 & & & & 0.3999 \\
\hline & $A p$ & on & 0.6800 & 0.8000 & 1.2000 \\
\hline
\end{tabular}

Exact and approximate solutions are calculated from Eqs. (30) and (31), respectively.

where $\varepsilon=1-(a / b)$. The values of the dimensionless drag force $-F / 6 \pi \eta b U$ calculated from this approximate formula are also listed in Table 2 for comparison. It can be found that the solution correct to the first order in $\varepsilon$ given by Eq. (31) agrees well with our collocation solutions for small magnitudes of $\varepsilon$. The errors are less than $4 \%$ for particles with $1<a / b<1.1$. However, the accuracy of this approximate solution begins to deteriorate, as expected, when the value of $a / b$ becomes greater, especially for the case of a perfectly slip spheroid.

The dimensionless drag force $-F / 6 \pi \eta b U$ for the axisymmetric motion of a prolate spheroid as a function of the aspect ratio $a / b$ for several different values of the slip parameter $\beta b / \eta$ is plotted in Fig. 2. For a prolate spheroid with a no-slip surface or a slip surface having large values of $\beta b / \eta$ (greater than about 1), as expected, the value of $-F / 6 \pi \eta b U$ increases monotonically with an increase in the value of $a / b$. For a slip prolate spheroid with a small value of $\beta b / \eta$ (less than about 0.1 ), however, $-F / 6 \pi \eta b U$ is a monotonically decreasing function of $a / b$. This behavior is understandable since the major portion of the fluid slip at the particle surface occurs in the direction of the particle's movement as the ratio $a / b$ is large. Note that, for a spheroid with values of $\beta b / \eta$ in the intermediate range (say, equal to 0.3), the dimensionless drag on it is not a monotonic function of $a / b$. When the ratio $a / b$ is large, the effect of the increase in the surface area (which experiences the hydrodynamic drag) of the prolate spheroid in comparison with a sphere of radius $b$ dominates, and the dimensionless drag increases with an increase in $a / b$. When 


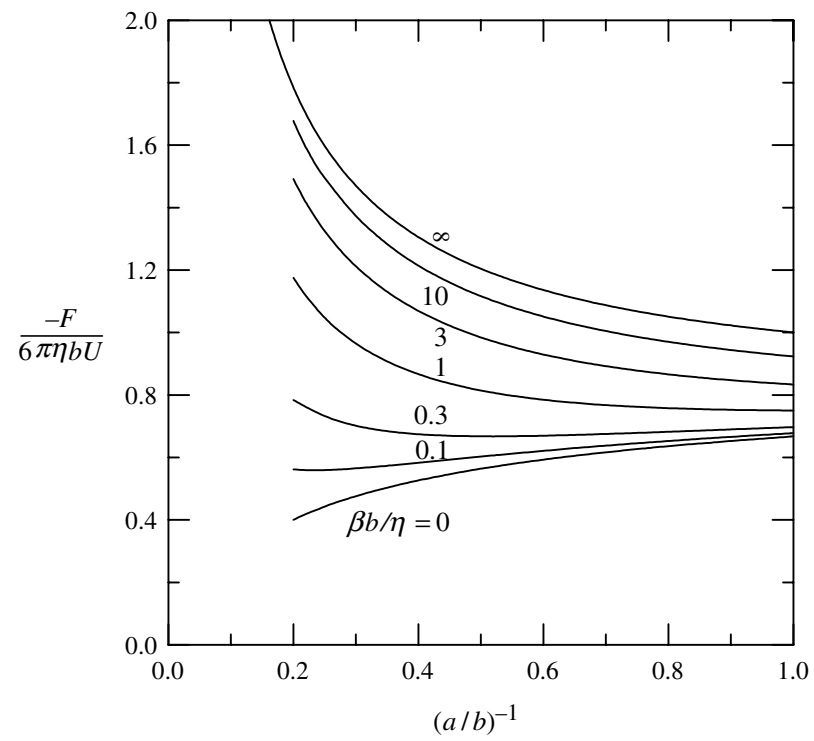

Fig. 2. Plots of the dimensionless drag force for the motion of a prolate spheroid with a slip surface along its axis of revolution versus the aspect ratio of the spheroid for various values of the slip parameter $\beta b / \eta$.

the ratio $a / b$ is close to unity, the effect of the fluid slip at the particle surface becomes more significant, and the dimensionless drag decreases with an increase in $a / b$. On the other hand, $-F / 6 \pi \eta b U$ is a monotonically increasing function of $\beta b / \eta$ for a given shape of spheroids, and its dependence becomes quite sensitive when the value of $a / b$ is large. It can be seen that the drag force on the spheroid can be very large when both $a / b$ and $\beta b / \eta$ are large.

\section{Axisymmetric motion of oblate particles}

The axisymmetric motion of a general prolate particle with a slip surface was considered in Section 4 and a set of Sampson spherical singularities must be distributed on a segment along the axis of symmetry inside the particle. In this section we consider the corresponding motion of an arbitrary slip oblate particle and the Sampsonlets should be distributed on the fundamental surface within the particle. Since the oblate particle and the fluid motion are axisymmetric, the fundamental surface should be a circular disk $S_{d}$ normal to the $z$-axis and with its center at the origin of the coordinate frame (the center of the particle).

Let Q be an arbitrary point on $S_{d}$ which is expressed with the circular polar coordinates $\rho=\hat{\rho}$, $\phi=\hat{\phi}$, and $z=0$. Then the velocity disturbance at another point $\mathrm{P}(\rho=\rho, \phi=0, z=z)$ generated by the Sampson singularity at $\mathrm{Q}$ can be obtained using Eq. (9a) and (9b),

$$
\hat{v}_{\rho}=\frac{\rho-\hat{\rho} \cos \hat{\phi}}{\rho^{*}} \sum_{n=2}^{\infty}\left[B_{n} A_{1 n}\left(\rho^{*}, z\right)+D_{n} A_{2 n}\left(\rho^{*}, z\right)\right]
$$




$$
\begin{aligned}
& \hat{v}_{\phi}=\frac{\hat{\rho} \sin \hat{\phi}}{\rho^{*}} \sum_{n=2}^{\infty}\left[B_{n} A_{1 n}\left(\rho^{*}, z\right)+D_{n} A_{2 n}\left(\rho^{*}, z\right)\right], \\
& \hat{v}_{z}=\sum_{n=2}^{\infty}\left[B_{n} C_{1 n}\left(\rho^{*}, z\right)+D_{n} C_{2 n}\left(\rho^{*}, z\right)\right],
\end{aligned}
$$

where $\rho^{*}$ is the distance from point $\mathrm{Q}$ to the projection of point $\mathrm{P}$ on the plane $z=0$,

$$
\rho^{*}=\left(\rho^{2}+\hat{\rho}^{2}-2 \rho \hat{\rho} \cos \hat{\phi}\right)^{1 / 2} \text {. }
$$

Due to the axisymmetry of the fluid motion, the singularities must be distributed uniformly on the circles in $S_{d}$ with their centers at the origin of coordinates. Hence, the unknown density distribution coefficients $B_{n}$ and $D_{n}$ in Eq. (32) are functions of $\hat{\rho}$ only.

The total disturbance of the flow field produced by the oblate particle can be approximated by the superposition of the individual disturbances in Eq. (32) induced by the whole set of singularities on the fundamental disk $S_{d}$. Thus, at an arbitrary location, we have

$$
\begin{aligned}
& v_{\rho}=\sum_{n=2}^{\infty} \int_{0}^{2 \pi} \int_{0}^{R} \frac{\rho-\hat{\rho} \cos \hat{\phi}}{\rho^{*}}\left[B_{n}(\hat{\rho}) A_{1 n}\left(\rho^{*}, z\right)+D_{n}(\hat{\rho}) A_{2 n}\left(\rho^{*}, z\right)\right] \hat{\rho} \mathrm{d} \hat{\rho} \mathrm{d} \hat{\phi} \\
& v_{z}=\sum_{n=2}^{\infty} \int_{0}^{2 \pi} \int_{0}^{R}\left[B_{n}(\hat{\rho}) C_{1 n}\left(\rho^{*}, z\right)+D_{n}(\hat{\rho}) C_{2 n}\left(\rho^{*}, z\right)\right] \hat{\rho} \mathrm{d} \hat{\rho} \mathrm{d} \hat{\phi}
\end{aligned}
$$

where $R$ is the radius of the disk $S_{d}$. The integral of $\hat{v}_{\phi}$ vanishes because the fluid motion is axisymmetric. Eq. (34) provides an exact solution for Eq. (3) that satisfies Eq. (7b), and the unknown density distribution functions $B_{n}(\hat{\rho})$ and $D_{n}(\hat{\rho})$ must be determined from the remaining boundary condition (7a) or (10) using the collocation method. In Eq. (10), the stress components can be calculated from Eq. (34) and expressed as

$$
\begin{aligned}
\tau_{\rho \rho}= & \eta \sum_{n=2}^{\infty} \int_{0}^{2 \pi} \int_{0}^{R}\left\{\frac{2 \hat{\rho}^{2} \sin ^{2} \hat{\phi}}{\left(\rho^{*}\right)^{3}}\left[B_{n}(\hat{\rho}) A_{1 n}\left(\rho^{*}, z\right)+D_{n}(\hat{\rho}) A_{2 n}\left(\rho^{*}, z\right)\right]\right. \\
& \left.+\left(\frac{\rho-\hat{\rho} \cos \hat{\phi}}{\rho^{*}}\right)^{2}\left[B_{n}(\hat{\rho}) \alpha_{1 n}\left(\rho^{*}, z\right)+D_{n}(\hat{\rho}) \alpha_{2 n}\left(\rho^{*}, z\right)\right]\right\} \hat{\rho} \mathrm{d} \hat{\rho} \mathrm{d} \hat{\phi} \\
\tau_{z z}= & \eta \sum_{n=2}^{\infty} \int_{0}^{2 \pi} \int_{0}^{R}\left[B_{n}(\hat{\rho}) \beta_{1 n}\left(\rho^{*}, z\right)+D_{n}(\hat{\rho}) \beta_{2 n}\left(\rho^{*}, z\right)\right] \hat{\rho} \mathrm{d} \hat{\rho} \mathrm{d} \hat{\phi}, \\
\tau_{\rho z}= & \eta \sum_{n=2}^{\infty} \int_{0}^{2 \pi} \int_{0}^{R} \frac{\rho-\hat{\rho} \cos \hat{\phi}}{\rho^{*}}\left[B_{n}(\hat{\rho}) \gamma_{1 n}\left(\rho^{*}, z\right)+D_{n}(\hat{\rho}) \gamma_{2 n}\left(\rho^{*}, z\right)\right] \hat{\rho} \mathrm{d} \hat{\rho} \mathrm{d} \hat{\phi} .
\end{aligned}
$$


The drag force exerted by the fluid on the oblate axisymmetric particle can be obtained by substituting Eq. (34) into Eq. (8) and applying the orthogonality properties of the Gegenbauer polynomials. The result is

$$
F=8 \pi^{2} \eta \int_{0}^{R} D_{2}(\hat{\rho}) \hat{\rho} \mathrm{d} \hat{\rho}
$$

Similar to the case of the motion of a prolate particle examined in Section 4, the density distribution functions $B_{n}(\hat{\rho})$ and $D_{n}(\hat{\rho})$ in Eqs. (34) and (35) can be approximated to various orders to satisfy the boundary condition (7a) or (10). In order to use the boundary-collocation method, the infinite series in Eqs. (34) and (35) are truncated after $N$ terms. The radius of the fundamental disk $S_{d}$ is divided into $M$ segments, and $\hat{\rho}_{m-1}$ and $\hat{\rho}_{m}$ denote the radial coordinates of the inner and outer end points, respectively, for the $m$ th segment. If the length of each segment is the same, then $\hat{\rho}_{j}=j R / M$ for $j=0,1,2, \ldots$, and $M$.

In the constant density approximation, the density distribution functions $B_{n}(\hat{\rho})$ and $D_{n}(\hat{\rho})$ in each segment are substituted by constants and the truncated form of Eqs. (34) and (35) can still be expressed by Eqs. (17) and (18) with functions $A_{i n m 1}, C_{i n m 1}, \alpha_{i n m 1}, \beta_{i n m 1}$, and $\gamma_{i n m 1}$ being replaced by $A_{i n m 1}^{\prime}, C_{i n m 1}^{\prime}, \alpha_{i n m 1}^{\prime}, \beta_{i n m 1}^{\prime}$, and $\gamma_{i n m 1}^{\prime}$ defined by Eqs. (A.9)-(A.11) in Appendix A, respectively. Thus, the collocation technique described in Section 4 can be used to satisfy the boundary condition (7a) and to determine the $2 M N$ density constants $B_{n m}$ and $D_{n m}$ required for the fluid velocity field. Since the density functions in each segment are constant, Eq. (36) for the drag force exerted on the oblate particle becomes

$$
F=4 \pi^{2} \eta \sum_{m=1}^{M} D_{2 m}\left(\hat{\rho}_{m}^{2}-\hat{\rho}_{m-1}^{2}\right)
$$

When the linear density distribution of Sampsonlets is employed, the distribution functions $B_{n}(\hat{\rho})$ and $D_{n}(\hat{\rho})$ in the $m$ th segment are given by Eq. (21) with $t, t_{m-1}$ and $t_{m}$ being replaced by $\hat{\rho}$, $\hat{\rho}_{m-1}$ and $\hat{\rho}_{m}$, respectively. Hence, the fluid velocity and stress components have the same form as Eqs. (22) and (23), but the functions $A_{i n m k}, C_{i n m k}, \alpha_{i n m k}, \beta_{i n m k}$, and $\gamma_{i n m k}$ in Eqs. (A.3) and (A.4) must be replaced by $A_{i n m k}^{\prime}, C_{i n m k}^{\prime}, \alpha_{i n m k}^{\prime}, \beta_{i n m k}^{\prime}$, and $\gamma_{i n m k}^{\prime}$, respectively. Application of the boundary condition (10) to these velocity and stress components can be accomplished by using the collocation method. After determining the $2(M+1) N$ unknown constants $B_{n m}$ and $D_{n m}$, the drag force exerted on the particle by the fluid can be evaluated by the formula

$$
F=\frac{4}{3} \pi^{2} \eta \sum_{m-1}^{M}\left[\left(\hat{\rho}_{m}+2 \hat{\rho}_{m-1}\right) D_{2(m-1)}+\left(2 \hat{\rho}_{m}+\hat{\rho}_{m-1}\right) D_{2 m}\right]\left(\hat{\rho}_{m}-\hat{\rho}_{m-1}\right),
$$

which is obtained by the substitution of $D_{2}(\hat{\rho})$ into Eq. (36).

In the quadratic density approximation, the distribution functions $B_{n}(\hat{\rho})$ and $D_{n}(\hat{\rho})$ in the $m$ th segment are given by Eq. (25) with $t, t_{m-1}, t_{m}$, and $\bar{t}_{m}$ being replaced by $\hat{\rho}, \hat{\rho}_{m-1}, \hat{\rho}_{m}$, and $\bar{\rho}_{m}\left(=\left(\hat{\rho}_{m-1}+\hat{\rho}_{m}\right) / 2\right)$, respectively, and the fluid velocity and stress components can also be written in the form of Eqs. (26) and (27) (after the substitution of $V_{i n m k}^{\prime}, W_{i n m k}^{\prime}, R_{i n m k}^{\prime}, S_{i n m k}^{\prime}$, and $T_{i n m k}^{\prime}$, defined by Eqs. (A.5)-(A.7)) with $A_{i n m k}, C_{i n m k}, \alpha_{i n m k}, \beta_{i n m k}$, and $\gamma_{i n m k}$ being replaced by $A_{i n m k}^{\prime}, C_{i n m k}^{\prime}, \alpha_{i n m k}^{\prime}, \beta_{i n m k}^{\prime}$, 
and $\gamma_{\text {inmk }}^{\prime}$, respectively. To apply the boundary condition $(10)$ to determine the $2(2 M+1) N$ unknown constants $B_{n m}$ and $D_{n m}$ required for the velocity field, the collocation technique must be used. Substituting $D_{2}(\hat{\rho})$ into Eq. (36), one obtains the drag force on the particle as

$$
F=\frac{4}{3} \pi^{2} \eta \sum_{m=1}^{M}\left[\hat{\rho}_{m-1} D_{2(2 m-2)}+2\left(\hat{\rho}_{m}+\hat{\rho}_{m-1}\right) D_{2(2 m-1)}+\hat{\rho}_{m} D_{2(2 m)}\right]\left(\hat{\rho}_{m}-\hat{\rho}_{m-1}\right) .
$$

Analogous to the case of a translating prolate particle, the accuracy and convergence behavior of the drag solution in Eqs. (37)-(39) depends on the precision of the numerical integrations of the functions $A_{i n m k}^{\prime}, C_{i n m k}^{\prime}, \alpha_{i n m k}^{\prime}, \beta_{i n m k}^{\prime}$, and $\gamma_{i n m k}^{\prime}$ defined by Eqs. (A.9)-(A.11).

\section{Solution for the motion of an oblate spheroidal particle}

In Section 5, numerical solutions of the drag force experienced by a prolate spheroid translating axisymmetrically were presented. In this section the similar singularity method and collocation technique described in the previous section will be used to solve the corresponding motion of an oblate spheroid. The shape of the oblate spheroid can still be represented by Eq. (29), but now with $b>a>0$. In addition, the exact solution for the drag force exerted on an oblate spheroid with a no-slip surface moving with a velocity $U$ along its axis of symmetry in an unbounded fluid can be expressed as Eq. (30a), with the coefficient $f_{\infty}$ given by [16]

$$
f_{\infty}=\left\{\frac{3}{4}\left(\zeta^{2}+1\right)^{1 / 2}\left[\zeta-\left(\zeta^{2}-1\right) \cot ^{-1} \zeta\right]\right\}^{-1},
$$

where $\zeta=a / c$ and $c=\left(b^{2}-a^{2}\right)^{1 / 2}$, which is the radius of the focal circle of the oblate spheroid.

The numerical solutions of the nondimensional drag force $-F / 6 \pi \eta b U$ for the axisymmetric motions of an oblate spheroid are presented in Table 3 for three representative cases of the aspect ratio $a / b$ with various values of the slip parameter $\beta b / \eta$. Either the linear (for $a / b=0.9$ and 0.5 ) or the quadratic (for $a / b=0.2$ ) density approximation is used to calculate the values of $F$ and to show the convergence tests. The exact solution for the motion of a no-slip oblate spheroid along its axis of revolution given by Eq. (40) and the approximate solution for the axisymmetric motion of a slip spheroid whose shape deviates slightly from that of a sphere given by Eq. (31) are also listed in Table 3 for comparison. Analogous to the case of a prolate spheroid considered in Section 5, the convergence behavior of the method of Sampson singularities in general is satisfactory. The agreement between our results and the exact and approximate solutions is quite good. The errors of Eq. (31) are less than 4\% for particles with $0.9<a / b<1$; but as expected, the accuracy of this approximate solution begins to deteriorate when the value of $a / b$ becomes smaller.

In Fig. 3, the dimensionless drag force $-F / 6 \pi \eta b U$ for the axisymmetric motion of an oblate spheroid as a function of the aspect ratio $a / b$ for several different values of the slip parameter $\beta b / \eta$ is plotted. Similarly to the motion of a prolate spheroid discussed in Section 5, the value of $-F / 6 \pi \eta b U$ increases monotonically as the ratio $a / b$ increases for an oblate spheroid with a noslip surface or a slip surface having large values of $\beta b / \eta$ (greater than about 10), and decreases 
Table 3

Numerical results of the dimensionless drag force for the motion of an oblate spheroid along its axis of revolution for various values of the aspect ratio and slip parameter of the spheroid

\begin{tabular}{|c|c|c|c|c|c|}
\hline \multirow[t]{2}{*}{$\beta b / \eta$} & \multirow[t]{2}{*}{$M$} & \multirow[t]{2}{*}{$N$} & \multicolumn{3}{|c|}{$-F / 6 \pi \eta b U$} \\
\hline & & & $a / b=0.9$ & $a / b=0.5$ & $a / b=0.2$ \\
\hline \multirow[t]{4}{*}{$\infty$} & 4 & 4 & 0.9801 & 0.9053 & 0.8614 \\
\hline & & 5 & 0.9801 & 0.9053 & 0.8615 \\
\hline & & 6 & 0.9801 & 0.9053 & 0.8615 \\
\hline & Exa & & 0.9801 & 0.9053 & 0.8615 \\
\hline \multirow[t]{4}{*}{10} & 4 & 4 & 0.9052 & 0.8448 & 0.8316 \\
\hline & & 5 & 0.9052 & 0.8448 & 0.8316 \\
\hline & & 6 & 0.9052 & 0.8448 & 0.8316 \\
\hline & Ap & & 0.9034 & 0.8249 & 0.7659 \\
\hline \multirow[t]{4}{*}{1} & 4 & 4 & 0.7496 & 0.7696 & 0.8157 \\
\hline & & 5 & 0.7496 & 0.7696 & 0.8157 \\
\hline & & 6 & 0.7496 & 0.7696 & 0.8157 \\
\hline & Ap & & 0.7338 & 0.6688 & 0.6200 \\
\hline \multirow[t]{4}{*}{0} & 4 & 4 & 0.6804 & 0.7470 & 0.8126 \\
\hline & & 5 & 0.6804 & 0.7470 & 0.8122 \\
\hline & & 6 & 0.6804 & 0.7470 & 0.8123 \\
\hline & Ap & & 0.6533 & 0.6000 & 0.5600 \\
\hline
\end{tabular}

Exact and approximate solutions are calculated from Eqs. (40) and (31), respectively.

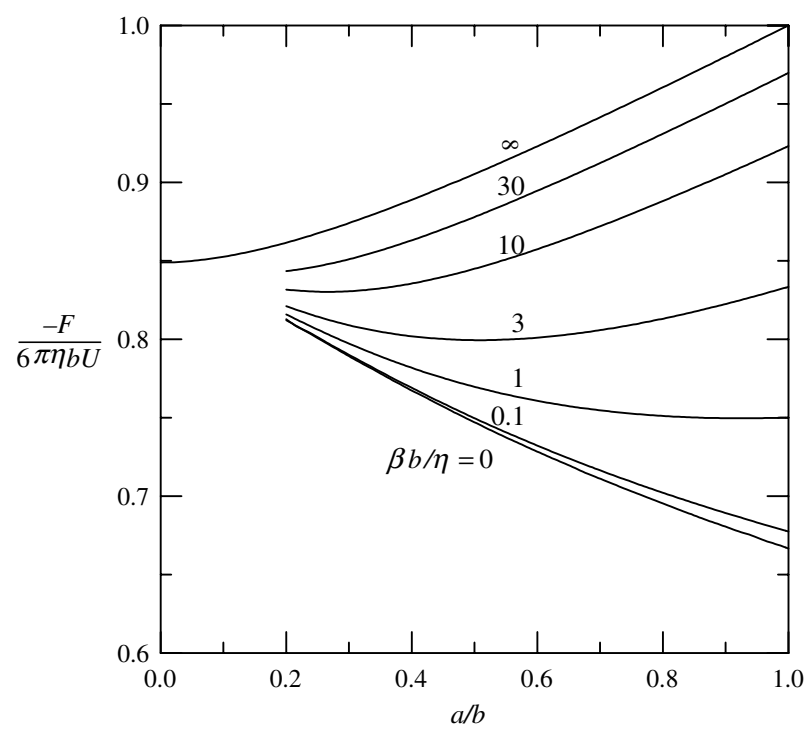

Fig. 3. Plots of the dimensionless drag force for the motion of an oblate spheroid with a slip surface along its axis of revolution versus the aspect ratio of the spheroid for various values of the slip parameter $\beta b / \eta$. 
monotonically with an increase in $a / b$ for a slip oblate spheroid with small values of $\beta b / \eta$ (less than about 1). The latter behavior is understandable since the main component of the fluid slip at the particle surface is in the direction normal to the motion of the spheroid when the value of $a / b$ becomes small. Again, $-F / 6 \pi \eta b U$ is not a monotonic function of $a / b$ for slip spheroids with a constant value of $\beta b / \eta$ in the intermediate range (say, equal to 3 ) and is a monotonically increasing function of $\beta b / \eta$ for spheroids with a fixed value of $a / b$.

Examination of the curves given in Figs. 2 and 3 reveals an interesting feature. For the translation of a slip spheroid with $\beta b / \eta$ equal to about unity, the normalized drag force on it decreases with an increase in $a / b$ (the effect of the fluid slip at the particle surface dominates) from $a / b \rightarrow 0$, reaches a minimum in the vicinity of $a / b=1$, and then increases with an increase in $a / b$ (the effect of the increase in the surface area of the particle dominates) until $a / b \rightarrow \infty$. On the other hand, for a spheroid having a large or small value of $\beta b / \eta(>10$ or $<0.1)$, its normalized drag force is a monotonic function of $a / b$ in the entire range of $0<a / b<\infty$.

\section{Concluding remarks}

In this work the creeping motion of a general axisymmetric particle with a slip surface in a viscous fluid (e.g., a slightly rarefied gas) along its axis of revolution has been analyzed by the use of the method of internal singularity distributions combined with the boundary-collocation technique. For the case of the axisymmetric motion of a prolate particle, a truncated set of Sampson singularities is distributed along the axis; while for the case of an oblate particle, the Sampsonlets are placed on the fundamental disk of the particle. The results for the drag force exerted on the particle by the fluid indicate that the solution procedure converges rapidly and accurate solutions can be obtained for various cases of the particle shape. Although the numerical solutions were presented in the previous sections only for a sphere, a prolate spheroid and an oblate spheroid, the combined analytical and numerical technique utilized in this work can easily provide the hydrodynamic calculations for an axisymmetric particle of other shapes, such as a prolate or oblate Cassini oval [25-27].

If the nose and tail of an axisymmetric prolate particle are not round, for example, the particle is a long cylinder, or if an axisymmetric oblate particle does not have a smooth surface, for example, the particle is a short cylinder or a relatively thin disk, the problem of the motion of such a particle will be difficult to be solved by any analytical or semi-analytical solution technique, and it is certainly not the main target of the solution technique considered in this work. In principle, however, the present solution technique might be better than other ones for solving this kind of problems. The Sampson spherical singularities can still be distributed on a segment along the axis of revolution of the prolate particle or on a fundamental disk within the oblate particle. Obviously, the choices of the end points of the segment and the radius of the fundamental disk are somewhat indefinite for this case. One would expect that slightly different but reasonable choices of the end points of the segment or the radius of the fundamental disk may lead to almost the same convergent solution for a given problem. On the other hand, if an arbitrary axisymmetric oblate particle has asymmetric surfaces with respect to the plane $z=0$, the fundamental surface can still be a disk normal to the $z$-axis with its center at the origin. For this case, the radius of the fundamental disk should be chosen with a compromise of the different needs between the surfaces 
with $z>0$ and with $z<0$. Again, one would expect that slightly different but reasonable choices of the radius of the fundamental disk may result in almost the same convergent solution for a given problem of this kind.

Throughout this work we have only considered the translation of a particle axisymmetrically. However, the method of internal distribution of spherical singularities can easily be employed to analyze the axisymmetric rotation of a particle of revolution. For example, the numerical solutions for the Stokes flow generated by the rotation of a no-slip prolate spheroid about its axis of symmetry were obtained by Yan et al. [33] using the method of spherical singularities combined with the boundary-collocation technique. Moreover, the method of axisymmetric singularity distribution can also be utilized to investigate some three-dimensional (asymmetric) flows induced by the motion of a body of revolution, such as the translation of a prolate spheroid in an arbitrary direction with respect to its axis of symmetry in an unbounded fluid [34].

A different approach with continuous distributions of internal singularities was proposed by Chwang and $\mathrm{Wu}[8]$ in which the Stokeslets, rotlets, stresslets, potential doublets, and some higher-order poles were distributed in a prescribed manner along an axis of symmetry. This led to exact solutions to a number of exterior and interior Stokes flow problems involving no-slip spheres, prolate spheroids, and infinitely long circular cylinders, but the problems for axisymmetric prolate particles of other shapes and oblate particles are still remained unsolved. It appears that the slip-flow boundary condition at the particle surface given by Eq. (7a) involving the viscous stresses makes their approach more difficult to apply than the present method of distributions of Sampsonlets combined with the collocation technique.

It is generally recognized that the distributed internal singularity methods are more limited in their range of application than the boundary integral equation method, which is much more flexible in treating problems with complicated body shapes and boundaries. Nonetheless, the singularity method presented herein has several advantages: (i) no singular integrals appear, since the collocation points lie on the body surface and the singular points lie within; (ii) closed-form recurrence formulas for the integrals in the linear algebraic equations have been obtained for the case of a prolate axisymmetric body, eliminating the need for a numerical integration; and (iii) the method, when it can be used, has proved to be far more accurate and efficient than the boundary integral equation technique.

\section{Acknowledgement}

This research was supported by the National Science Council of the Republic of China.

\section{Appendix A}

For conciseness the definitions of some functions in Sections 2, 4 and 6 are listed in this appendix. The functions appearing in Eqs. (9) and (11) in Section 2 are defined as

$$
A_{1 n}(\rho, z)=(n+1) \rho^{-1}\left(\rho^{2}+z^{2}\right)^{-n / 2} G_{n+1}^{-1 / 2}(\mu),
$$




$$
\begin{aligned}
& A_{2 n}(\rho, z)=\rho^{-1}\left(\rho^{2}+z^{2}\right)^{-(n-2) / 2}\left[-2 \mu G_{n}^{-1 / 2}(\mu)+(n+1) G_{n+1}^{-1 / 2}(\mu)\right], \\
& C_{1 n}(\rho, z)=\left(\rho^{2}+z^{2}\right)^{-(n+1) / 2} P_{n}(\mu), \\
& C_{2 n}(\rho, z)=\left(\rho^{2}+z^{2}\right)^{-(n-1) / 2}\left[2 G_{n}^{-1 / 2}(\mu)+P_{n}(\mu)\right], \\
& E_{1 n}(\rho, z)=\left(\rho^{2}+z^{2}\right)^{-(n-1) / 2} G_{n}^{-1 / 2}(\mu), \\
& E_{2 n}(\rho, z)=\left(\rho^{2}+z^{2}\right)^{-(n-3) / 2} G_{n}^{-1 / 2}(\mu) ; \\
& \alpha_{1 n}(\rho, z)=-2(n+1) \rho^{-2}\left(\rho^{2}+z^{2}\right)^{-(n+2) / 2}\left[\left(n \rho^{2}+\rho^{2}+z^{2}\right) G_{n+1}^{-1 / 2}(\mu)-\rho^{2} \mu P_{n}(\mu)\right], \\
& \alpha_{2 n}(\rho, z)=2 \rho^{-2}\left(z^{2}+\rho^{2}\right)^{-n / 2}\left[2\left(n \rho^{2}+z^{2}\right) \mu G_{n}^{-1 / 2}(\mu)-(n+1)\left(n \rho^{2}-\rho^{2}+z^{2}\right) G_{n+1}^{-1 / 2}(\mu)\right. \\
& \left.-2 \rho^{2} z^{2}\left(\rho^{2}+z^{2}\right)^{-1} P_{n-1}(\mu)+(1+n) \rho^{2} \mu P_{n}(\mu)\right], \\
& \beta_{1 n}(\rho, z)=2\left(\rho^{2}+z^{2}\right)^{-(n+2) / 2}\left[n P_{n-1}(\mu)-(2 n+1) \mu P_{n}(\mu)\right], \\
& \beta_{2 n}(\rho, z)=2\left(\rho^{2}+z^{2}\right)^{-n / 2}\left[-2(n-1) \mu G_{n}^{-1 / 2}(\mu)+\left(\rho^{2}+z^{2}\right)^{-1}\left(n \rho^{2}-2 \rho^{2}+n z^{2}\right) P_{n-1}(\mu)\right. \\
& \left.-(2 n-1) \mu P_{n}(\mu)\right] \text {, } \\
& \gamma_{1 n}(\rho, z)=\rho^{-1}\left(\rho^{2}+z^{2}\right)^{-(n+1) / 2}\left[-n(n+1) \mu G_{n+1}^{-1 / 2}(\mu)-n \mu P_{n-1}(\mu)-\left(\rho^{2}+z^{2}\right)^{-1}\right. \\
& \left.\times\left(2 n \rho^{2}+2 \rho^{2}-n z^{2}\right) P_{n}(\mu)\right], \\
& \gamma_{2 n}(\rho, z)=\rho^{-1}\left(\rho^{2}+z^{2}\right)^{-(n+1) / 2}\left[-2\left(n \rho^{2}-n z^{2}+2 z^{2}\right) G_{n}^{-1 / 2}(\mu)-\left(n^{2}-n-2\right)\right. \\
& \left.\times\left(\rho^{2}+z^{2}\right) \mu G_{n+1}^{-1 / 2}(\mu)-\left(n \rho^{2}-4 \rho^{2}+n z^{2}\right) \mu P_{n-1}(\mu)-n\left(2 \rho^{2}-z^{2}\right) P_{n}(\mu)\right],
\end{aligned}
$$

where $\mu=z\left(\rho^{2}+z^{2}\right)^{-1 / 2}, G_{n}^{-1 / 2}$ is the Gegenbauer polynomial of the first kind of order $n$ and degree $-1 / 2$, and $P_{n}$ is the Legendre polynomial of order $n$.

The functions appearing in Eqs. (22), (23), (26) and (27) in Section 4 are defined as

$$
\begin{aligned}
& {\left[\begin{array}{c}
V_{i n m 1}(\rho, z) \\
W_{i n m 1}(\rho, z) \\
R_{i n m 1}(\rho, z) \\
S_{i n m 1}(\rho, z) \\
T_{i n m 1}(\rho, z)
\end{array}\right]=\frac{1}{t_{m}-t_{m-1}}\left\{t_{m}\left[\begin{array}{c}
A_{i n m 1}(\rho, z) \\
C_{i n m 1}(\rho, z) \\
\alpha_{i n m 1}(\rho, z) \\
\beta_{i n m 1}(\rho, z) \\
\gamma_{i n m 1}(\rho, z)
\end{array}\right]-\left[\begin{array}{c}
A_{i n m 2}(\rho, z) \\
C_{i n m 2}(\rho, z) \\
\alpha_{i n m 2}(\rho, z) \\
\beta_{i n m 2}(\rho, z) \\
\gamma_{i n m 2}(\rho, z)
\end{array}\right]\right\},} \\
& {\left[\begin{array}{l}
V_{\text {inm } 2}(\rho, z) \\
W_{\text {inm } 2}(\rho, z) \\
R_{\text {inm } 2}(\rho, z) \\
S_{\text {inm } 2}(\rho, z) \\
T_{\text {inm } 2}(\rho, z)
\end{array}\right]=\frac{-1}{t_{m}-t_{m-1}}\left\{t_{m-1}\left[\begin{array}{c}
A_{\text {inm } 1}(\rho, z) \\
C_{\text {inm } 1}(\rho, z) \\
\alpha_{\text {inm } 1}(\rho, z) \\
\beta_{\text {inm } 1}(\rho, z) \\
\gamma_{\text {inm } 1}(\rho, z)
\end{array}\right]-\left[\begin{array}{c}
A_{\text {inm } 2}(\rho, z) \\
C_{\text {inm } 2}(\rho, z) \\
\alpha_{\text {inm } 2}(\rho, z) \\
\beta_{\text {inm } 2}(\rho, z) \\
\gamma_{\text {inm } 2}(\rho, z)
\end{array}\right]\right\},}
\end{aligned}
$$




$$
\begin{aligned}
& {\left[\begin{array}{c}
V_{i n m 1}^{\prime}(\rho, z) \\
W_{i n m 1}^{\prime}(\rho, z) \\
R_{i n m 1}^{\prime}(\rho, z) \\
S_{i n m 1}^{\prime}(\rho, z) \\
T_{i n m 1}^{\prime}(\rho, z)
\end{array}\right]=\frac{2}{\left(t_{m}-t_{m-1}\right)^{2}}\left\{t_{m} \bar{t}_{m}\left[\begin{array}{c}
A_{i n m 1}(\rho, z) \\
C_{i n m 1}(\rho, z) \\
\alpha_{i n m 1}(\rho, z) \\
\beta_{i n m 1}(\rho, z) \\
\gamma_{\text {inm } 1}(\rho, z)
\end{array}\right]-\left(t_{m}+\bar{t}_{m}\right)\left[\begin{array}{c}
A_{\text {inm } 2}(\rho, z) \\
C_{i n m 2}(\rho, z) \\
\alpha_{i n m 2}(\rho, z) \\
\beta_{i n m 2}(\rho, z) \\
\gamma_{i n m 2}(\rho, z)
\end{array}\right]\right.} \\
& \left.+\left[\begin{array}{c}
A_{\text {inm } 3}(\rho, z) \\
C_{\text {inm } 3}(\rho, z) \\
\alpha_{\text {inm } 3}(\rho, z) \\
\beta_{\text {inm } 3}(\rho, z) \\
\gamma_{\text {inm } 3}(\rho, z)
\end{array}\right]\right\}
\end{aligned}
$$

$$
\begin{gathered}
{\left[\begin{array}{c}
V_{\text {inm } 2}^{\prime}(\rho, z) \\
W_{i n m 2}^{\prime}(\rho, z) \\
R_{\text {inm } 2}^{\prime}(\rho, z) \\
S_{\text {inm } 2}^{\prime}(\rho, z) \\
T_{\text {inm } 2}^{\prime}(\rho, z)
\end{array}\right]=\frac{-4}{\left(t_{m}-t_{m-1}\right)^{2}}\left\{t_{m} t_{m-1}\left[\begin{array}{c}
A_{\text {inm } 1}(\rho, z) \\
C_{i n m 1}(\rho, z) \\
\alpha_{i n m 1}(\rho, z) \\
\beta_{i n m 1}(\rho, z) \\
\gamma_{i n m 1}(\rho, z)
\end{array}\right]-\left(t_{m}+t_{m-1}\right)\left[\begin{array}{l}
A_{\text {inm } 2}(\rho, z) \\
C_{i n m 2}(\rho, z) \\
\alpha_{i n m 2}(\rho, z) \\
\beta_{i n m 2}(\rho, z) \\
\gamma_{i n m 2}(\rho, z)
\end{array}\right]\right.} \\
\left.+\left[\begin{array}{c}
A_{i n m 3}(\rho, z) \\
C_{i n m 3}(\rho, z) \\
\alpha_{i n m 3}(\rho, z) \\
\beta_{i n m 3}(\rho, z) \\
\gamma_{i n m 3}(\rho, z)
\end{array}\right]\right\}
\end{gathered}
$$

$$
\begin{gathered}
{\left[\begin{array}{c}
V_{i n m 3}^{\prime}(\rho, z) \\
W_{i n m 3}^{\prime}(\rho, z) \\
R_{i n m 3}^{\prime}(\rho, z) \\
S_{i n m 3}^{\prime}(\rho, z) \\
T_{i n m 3}^{\prime}(\rho, z)
\end{array}\right]=\frac{2}{\left(t_{m}-t_{m-1}\right)^{2}}\left\{t_{m-1} \bar{t}_{m}\left[\begin{array}{c}
A_{i n m 1}(\rho, z) \\
C_{i n m 1}(\rho, z) \\
\alpha_{i n m 1}(\rho, z) \\
\beta_{i n m 1}(\rho, z) \\
\gamma_{i n m 1}(\rho, z)
\end{array}\right]-\left(t_{m-1}+\bar{t}_{m}\right)\left[\begin{array}{l}
A_{\text {inm } 2}(\rho, z) \\
C_{i n m 2}(\rho, z) \\
\alpha_{i n m 2}(\rho, z) \\
\beta_{i n m 2}(\rho, z) \\
\gamma_{i n m 2}(\rho, z)
\end{array}\right]\right.} \\
\left.+\left[\begin{array}{c}
A_{i n m 3}(\rho, z) \\
C_{i n m 3}(\rho, z) \\
\alpha_{i n m 3}(\rho, z) \\
\beta_{i n m 3}(\rho, z) \\
\gamma_{i n m 3}(\rho, z)
\end{array}\right]\right\}
\end{gathered}
$$

where $m=1,2, \ldots, M, n=2,3, \ldots, N+1$, and $i=1,2$. In Eqs. (17) and (18), and (A.3)-(A.7), 


$$
\left[\begin{array}{c}
A_{\text {inmk }}(\rho, z) \\
C_{\text {inmk }}(\rho, z) \\
\alpha_{\text {inmk }}(\rho, z) \\
\beta_{\text {inmk }}(\rho, z) \\
\gamma_{\text {inmk }}(\rho, z)
\end{array}\right]=\int_{t_{m-1}}^{t_{m}}\left\{t^{k-1}\left[\begin{array}{c}
A_{\text {in }}(\rho, z-t) \\
C_{\text {in }}(\rho, z-t) \\
\alpha_{\text {in }}(\rho, z-t) \\
\beta_{\text {in }}(\rho, z-t) \\
\gamma_{\text {in }}(\rho, z-t)
\end{array}\right]\right\} \mathrm{d} t,
$$

where $k=1,2$, and 3. The above five integrals can be evaluated analytically using Eqs. (A.1) and (A.2).

The following are the definitions of some functions used in Section 6:

$$
\begin{aligned}
& {\left[\begin{array}{c}
A_{\text {inmk }}^{\prime}(\rho, z) \\
\gamma_{\text {inmk }}^{\prime}(\rho, z)
\end{array}\right]=\int_{0}^{2 \pi} \int_{\hat{\rho}_{m-1}}^{\hat{\rho}_{m}} \hat{\rho}^{k} \frac{\rho-\hat{\rho} \cos \hat{\phi}}{\rho^{*}}\left[\begin{array}{c}
A_{\text {in }}\left(\rho^{*}, z\right) \\
\gamma_{\text {in }}\left(\rho^{*}, z\right)
\end{array}\right] \mathrm{d} \hat{\rho} \mathrm{d} \hat{\phi},} \\
& {\left[\begin{array}{c}
C_{i n m k}^{\prime}(\rho, z) \\
\beta_{\text {inmk }}^{\prime}(\rho, z)
\end{array}\right]=\int_{0}^{2 \pi} \int_{\hat{\rho}_{m-1}}^{\hat{\rho}_{m}} \hat{\rho}^{k}\left[\begin{array}{c}
C_{i n}\left(\rho^{*}, z\right) \\
\beta_{i n}\left(\rho^{*}, z\right)
\end{array}\right] \mathrm{d} \hat{\rho} \mathrm{d} \hat{\phi},} \\
& \alpha_{i n m k}^{\prime}(\rho, z)=\int_{0}^{2 \pi} \int_{\hat{\rho}_{m-1}}^{\hat{\rho}_{m}} \hat{\rho}^{k}\left[\frac{2 \hat{\rho}^{2} \sin ^{2} \hat{\phi}}{\left(\rho^{*}\right)^{3}} A_{\text {in }}\left(\rho^{*}, z\right)+\left(\frac{\rho-\hat{\rho} \cos \hat{\phi}}{\rho^{*}}\right)^{2} \alpha_{i n}\left(\rho^{*}, z\right)\right] \mathrm{d} \hat{\rho} \mathrm{d} \hat{\phi},
\end{aligned}
$$

where $\rho^{*}$ is defined by Eq. (33), $n=2,3, \ldots, N+1, m=1,2, \ldots, M, i=1,2$, and $k=1,2,3$. The integrations in Eqs. (A.9)-(A.11) can be performed numerically after the substitution of Eqs. (A.1) and (A.2).

\section{References}

[1] G.G. Stokes, On the effect of the internal friction of fluid on pendulums, Trans. Cambridge Philos. Soc. 9 (1851) 8106.

[2] A. Oberbeck, Uber stationare Flussigkeitsbewegungen mit Berucksichtigung der inner Reibung, J. Reine Angew. Math. 81 (1876) 62-80.

[3] L.E. Payne, W.H. Pell, The Stokes flow problem for a class of axially symmetric bodies, J. Fluid Mech. 7 (1960) 529-549.

[4] S.L. Goren, M.E. O'Neill, Asymmetric creeping motion of an open torus, J. Fluid Mech. 101 (1980) 97-110.

[5] G.K. Batchelor, Slender-body theory for particles of arbitrary cross-section in Stokes flow, J. Fluid Mech. 44 (1970) 419-440.

[6] R.G. Cox, The motion of long slender bodies in a viscous fluid, Part 1. General theory, J. Fluid Mech. 44 (1970) 791-810.

[7] M.J. Gluckman, S. Weinbaum, R. Pfeffer, Axisymmetric slow viscous flow past an arbitrary convex body of revolution, J. Fluid Mech. 55 (1972) 677-709.

[8] A.T. Chwang, T.Y. Wu, Hydrodynamic of low-Reynolds-number flow, Part 2. Singularity method for Stokes flows, J. Fluid Mech. 67 (1975) 787-815.

[9] G.K. Youngren, A. Acrivos, Stokes flow past a particle of arbitrary shape: a numerical method of solution, J. Fluid Mech. 69 (1975) 377-403.

[10] S. Weinbaum, P. Ganatos, Z. Yan, Numerical multipole and boundary integral equation techniques in Stokes flow, Annu. Rev. Fluid Mech. 22 (1990) 275-316.

[11] C. Pozrikidis, Boundary Integral and Singularity Methods for Linearized Viscous Flow, Cambridge University Press, Cambridge, 1992. 
[12] E.H. Kennard, Kinetic Theory of Gases, McGraw-Hill, New York, 1938.

[13] R. Ying, M.H. Peters, Interparticle and particle-surface gas dynamic interactions, Aerosol Sci. Technol. 14 (1991) 418-433.

[14] D.K. Hutchins, M.H. Harper, R.L. Felder, Slip correction measurements for solid spherical particles by modulated dynamic light scattering, Aerosol Sci. Technol. 22 (1995) 202-218.

[15] A.B. Basset, A Treatise on Hydrodynamics, vol. 2, Dover, New York, 1961.

[16] J. Happel, H. Brenner, Low Reynolds Number Hydrodynamics, Nijhoff, The Netherlands, 1983.

[17] M.H. Davis, Collisions of small cloud droplets: gas kinetic effects, J. Atmospheric Sci. 29 (1972) 911-915.

[18] L. Talbot, R.K. Cheng, R.W. Schefer, D.R. Willis, Thermophoresis of particles in heated boundary layer, J. Fluid Mech. 101 (1980) 737-758.

[19] M.L. Laucks, G. Roll, G. Schweiger, E.J. Davis, Physical and chemical (Raman) characterization of bioaerosolspollen, J. Aerosol Sci. 31 (2000) 307-319.

[20] B.E. Dahneke, Slip correction factors for nonspherical bodies-III The form of the general law, J. Aerosol Sci. 4 (1973) $163-170$.

[21] D. Palaniappan, Creeping flow about a slightly deformed sphere, ZAMP 45 (1994) 832-838.

[22] H. Ramkissoon, Slip flow past an approximate spheroid, Acta Mechanica 123 (1997) 227-233.

[23] K.H. Leong, Thermophoresis and diffusiophoresis of large aerosol particles of different shapes, J. Aerosol Sci. 15 (1984) 511-517.

[24] M.M.R. Williams, Thermophoretic forces acting on a spheroid, J. Phys. D: Appl. Phys. 19 (1986) 1631-1642.

[25] W. Wu, A new approach of treating the Stokes flow of nonslender prolate arbitrary axisymmetrical body, Sci. Sin. Ser. A 27 (1984) 731-744.

[26] M. Zhu, W. Wu, The method of continuous distribution of singularities to treat the Stokes flow of the arbitrary oblate axisymmetrical body, Appl. Math. Mech. SUT, Shanghai, China 6 (1985) 929-942.

[27] H.J. Keh, C.H. Tseng, Slow motion of an arbitrary axisymmetric body along its axis of revolution and normal to a plane surface, Int. J. Multiphase Flow 20 (1994) 185-210.

[28] R.A. Sampson, On Stokes's current function, Philos. Trans. R. Soc. A 182 (1891) 449-518.

[29] V. O'Brien, Form factors for deformed spheroids in Stokes flow, AIChE J. 14 (1968) 870-875.

[30] M.J. Gluckman, R. Pfeffer, S. Weinbaum, A new technique for treating multi-particle slow viscous flow: axisymmetric flow past spheres and spheroids, J. Fluid Mech. 50 (1971) 705-740.

[31] H.J. Keh, L.C. Lien, Electrophoresis of a colloidal sphere along the axis of a circular orifice or a circular disk, J. Fluid Mech. 224 (1991) 305-333.

[32] J. Feng, W. Wu, Electrophoretic motion of an arbitrary prolate body of revolution toward an infinite conducting wall, J. Fluid Mech. 264 (1994) 41-58.

[33] Z. Yan, Y. Zhao, W. Liu, The Stokes flow induced by the rotation of a prolate axisymmetric body in the presence of an infinite planar boundary, in: H. Niki, M. Kawahara (Eds.), Computational Methods in Flow Analysis, vol. 1, Okayama University of Science, Okayama, Japan, 1988, pp. 243-248.

[34] S. Lin, W. Wu, The semi-analytic semi-numerical method to treat the three-dimension Stokes flow, Acta Aerodyn. Sin. 4 (1986) 140-149 (in Chinese). 